\title{
46. The Flat Holomorphic Conformal Structure on the Horrocks-Mumford Orbifold
}

\author{
By Takeshi SATo \\ Department of Mathematics, Faculty of Science, University of Tokyo \\ (Communicated by Kunihiko KodaIRA, M. J. A., May 13, 1991)
}

We construct the explicit flat holomorphic conformal structure on an orbifold. We often abbreviate 'Horrocks-Mumford' to 'HM', and 'holomorphic conformal structure' to 'HCS'. $P_{n}(C)$ denotes the $n$-dimensional complex projective space.

In the paper [2], Horrocks and Mumford constructed a holomorphic vector bundle $\mathscr{F}_{H M}$ of rank two on the $P_{4}(C)$. The space $\Gamma \mathscr{I}_{H M}$ of its sections is four-dimensional. If the zero set $X_{s}$ of a section $s \in \Gamma \mathscr{I}_{H M}$ is a smooth surface, $X_{s}$ is an abelian surface with (1.5)-polarization. In fact, they proved that $P_{3}(C)=P\left(\Gamma \mathscr{F}_{H M}\right)$ is birationally equivalent to the moduli space $\mathscr{A}_{1,5}$ of the abelian surfaces with $(1,5)$-polarization and level-5structure. (See [2] [4].) We call this projective space the HM-orbifold.

While the moduli space $\mathscr{A}_{1,5}$ is realized as a quotient space $\mathcal{H}_{2} / \Gamma_{1,5}$ of the Siegel upper space $\mathscr{H}_{2}$ of degree two. Here $\Gamma_{1,5}$ is a certain discrete subgroup of $S p(4, R)$. (See [4].) $\mathscr{H}_{2}$ is embedded in a non-degenerate hyperquadrics $\left\{\left[z_{0}: z_{1}: z_{2}: z_{3}: z_{4}\right] \in P_{4}(C) ; \sum_{0 \leqq i, j \leqq 4} a_{i j} z_{i} z_{j}=0\right\}$. The holomorphic tensor field $\phi=\sum_{0 \leqq i, j \leqq 4} a_{i j} d z_{i} d z_{j}$ on $\mathcal{H}_{2}$ is conformally flat and its conformally class is invariant under the automorphisms of $\mathcal{H}_{2}$. Therefore $\phi$ induces a tensor $\varphi$ on the HM-orbifold which is called the flat HCS. Applying a higher dimensional version of Kobayashi and Naruki's theory in [3], we can calculate the flat HCS.

Theorem 1. Let $p$ be the projection $C^{4} \backslash\{0\} \rightarrow P_{3}(C)$. The pullback of the flat $\mathrm{HCS} \varphi$ is given by in homogeneous coordinates

where

$$
p^{*} \varphi=\sum_{0 \leqq i, j \leqq 3} g_{i j} d x_{i} d x_{j}
$$

$$
\begin{aligned}
& g_{00}=2\left(-x_{0}^{2} x_{1} x_{2}-x_{0}^{2} x_{3}^{2}+x_{0} x_{1}^{3}+2 x_{0} x_{2}^{2} x_{3}+2 x_{1}^{2} x_{2} x_{3}-3 x_{1} x_{3}^{3}\right) \\
& g_{01}=x_{0}^{3} x_{2}-2 x_{0}^{2} x_{1}^{2}-7 x_{0} x_{1} x_{2} x_{3}+4 x_{0} x_{3}^{3}+x_{1}^{3} x_{3}+4 x_{1} x_{2}^{3}-5 x_{2}^{2} x_{3}^{2} \\
& g_{02}=x_{0}^{3} x_{1}-x_{0}^{2} x_{2} x_{3}-x_{0} x_{1}^{2} x_{3}-4 x_{1}^{2} x_{2}^{2}+5 x_{1} x_{2} x_{3}^{2} \\
& g_{03}=2 x_{0}^{3} x_{3}-3 x_{0}^{2} x_{2}^{2}+4 x_{0} x_{1}^{2} x_{2}+2 x_{0} x_{1} x_{3}^{2}-x_{1}^{4} \\
& g_{11}=2\left(x_{0}^{3} x_{1}+2 x_{0}^{2} x_{2} x_{3}-x_{0} x_{1}^{2} x_{3}-3 x_{0} x_{2}^{3}-x_{1}^{2} x_{2}^{2}+2 x_{1} x_{2} x_{3}^{2}\right) \\
& g_{12}=-x_{0}^{4}+4 x_{0}^{2} x_{1} x_{3}+2 x_{0} x_{1} x_{2}^{2}+2 x_{1}^{3} x_{2}-3 x_{1}^{2} x_{3}^{2} \\
& g_{13}=-x_{0}^{2} x_{1} x_{2}-4 x_{0}^{2} x_{3}^{2}+x_{0} x_{1}^{3}+5 x_{1} x_{2}^{2} x_{3}-x_{1}^{2} x_{2} x_{3} \\
& g_{22}=2\left(-x_{0}^{3} x_{3}+x_{0} x_{1}^{2} x_{2}+5 x_{0} x_{1} x_{3}^{2}-x_{1}^{4}\right) \\
& g_{23}=3\left(x_{0}^{3} x_{2}-x_{0}^{2} x_{1}^{2}-5 x_{0} x_{1} x_{2} x_{3}+x_{1}^{3} x_{3}\right) \\
& g_{33}=2\left(-x_{0}^{4}+x_{0}^{2} x_{1} x_{3}+5 x_{0} x_{1} x_{2}^{2}-x_{1}^{3} x_{2}\right) \\
& g_{10}=g_{01}, g_{20}=g_{02}, g_{30}=g_{03}, g_{21}=g_{12}, g_{31}=g_{13}, g_{32}=g_{23} .
\end{aligned}
$$


This flat HCS is degenerate along the trisecant surface to the rational sextic curve $C:\left(5 \lambda^{4}: 5 \lambda^{2}: \lambda^{6}-2 \lambda: 2 \lambda^{5}+1\right)$. (See [1].)

Remark. A Hilbert modular surface for $Q(\sqrt{5})$ is embedded in the HM-orbifold as the diagonal cubic of Clebsch. Pullback of the flat HCS to the cubic surface gives the HCS obtained in $[3,(6.3)]$.

We quote a theorem from [5].

Theorem 2 ([5, Theorem 2.5]). Assume the dimension of the space = $n \geqq 3$. Let $\sum \sigma_{i j} d x^{i} d x^{j}$ be a non-degenerate symmetric tensor which is conformally flat. Then the system

$$
\sigma_{i j}\left(w_{k l}-\sum_{p} \Gamma_{k l}^{p} w_{p}+\frac{1}{n-2} R_{k l} w\right)=\sigma_{k l}\left(w_{i j}-\sum_{p} \Gamma_{i j}^{p} w_{p}+\frac{1}{n-2} R_{i j} w\right)
$$

is of rank $n+2$ and ratio $\left[s_{0}: \cdots: s_{n+1}\right]$ of its linearly independent solutions takes its values in a hyperquadrics. Here $\Gamma_{j k}^{i}$ and $R_{i j}$ stand for the Christoffel symbol and the Ricci tensor with respect to $\sigma_{i j}$ and $w_{i}$ is the derivative of $w$ with respect to $x_{i}$.

As a corollary, we obtain the explicit form of the uniformizing differential equation of the HM-orbifold in the sense of Yoshida [6]. However, we have to omit the Christoffel symbol and the Ricci tensor with respect to the flat HCS $g_{i j}$ because they are far from simple.

\section{References}

[1] W. Barth and R. Moore: Geometry in the space of Horrocks-Mumford surfaces. Topology, 28, 231-345 (1989).

[2] G. Horrocks and D. Mumford: A rank 2 vector bundle on $\boldsymbol{P}^{4}$ with 15,000 symmetries. ibid., 12, 63-81 (1973).

[3] R. Kobayashi and I. Naruki: Holomorphic conformal structures and uniformization of complex surfaces. Math. Ann., 279, 485-500 (1988).

[4] K. Hulek and H. Lange: The Hilbert modular surface for the ideal $(\sqrt{5})$ and the Horrocks-Mumford bundle. Math. Z., 198, 95-116 (1988).

[5] T. Sasaki and M. Yoshida: Linear differential equations modeled after hyperquadrics. Tôhoku Math. J., 41, 321-348 (1989).

[6] M. Yoshida: Fuchsian Differential Equations. Aspects of Math., Vieweg Verlag, Weisbarden (1987). 
